\title{
Development of a novel ex vivo equine corneal model
}

\author{
Todd L Marlo ${ }^{1}$, Elizabeth A Giuliano ${ }^{1,2}$, Ajay Sharma ${ }^{1,2, \$}$, and Rajiv R Mohan ${ }^{1,2,3}$ \\ ${ }^{1}$ Department of Veterinary Medicine and Surgery, College of Veterinary Medicine, University of \\ Missouri, Columbia, MO \\ ${ }^{2}$ Harry S. Truman Veterans Memorial Hospital, Columbia, MO \\ ${ }^{3}$ Mason Eye Institute, University of Missouri, 1 Hospital Drive, Columbia, MO 65212
}

\begin{abstract}
Objective-To develop an ex vivo equine corneal organ culture model. Specifically, to assess the equine cornea's extracellular matrix and cellularity after 7 days using two different culture techniques: either (a) immersion system or (b) air/liquid interface system, to determine the best $e x$ vivo equine corneal model.
\end{abstract}

Methods-Fourteen healthy equine corneas with $2 \mathrm{~mm}$ of perilimbal sclera were freshly harvested from 7 horses undergoing humane euthanasia. One corneal-scleral ring (CSR) from each horse was randomly placed in the (a) immersion condition organ culture system (IC), with the contralateral SCR being placed in the (b) air/liquid interface organ culture system (ALC) for 7 days. All corneas were evaluated using serial daily gross photography, histology, qPCR and TUNEL assay.

Results-CSRs placed in the IC (a) had complete loss of corneal transparency on gross photography by 7 days, showed a significant level of corneal stromal disorganization, significantly increased aSMA levels on qPCR, and apoptosis on TUNEL assay compared to controls. The ALC (b), had weak stromal disorganization on histopathologic examination and was not significantly different from normal equine corneal controls on all other evaluated parameters.

Conclusions-The air-liquid interface organ culture system maintains the equine cornea's extracellular matrix and preserves corneal transparency, while the immersion condition results in near complete degradation of normal equine corneal architecture after 7 days in culture. The airliquid organ culture is a viable option to maintain a healthy equine cornea in an ex-vivo setting for wound healing studies.

\section{Keywords}

Cornea; Ex Vivo; Equine; Model; Transparency; Extracellular matrix

Address communication to: Rajiv R Mohan, 1600 E. Rollins Rd., University of Missouri, Columbia MO 65211. Telephone:

1-573-884-1449, Fax: 1-573-884-4100, MohanR@ health.missouri.edu.

Present address: Harry and Diane Rinker Health Science Campus, Chapman University, Irvine, CA 92618 


\section{Introduction}

Corneal disease in horses includes ulcerative, fungal, traumatic, and neoplastic conditions [1]. While these diseases are currently treated with a variety of medical and surgical modalities, permanent vision loss commonly results due to extensive corneal fibrosis.

Equine corneal research to date has typically been performed using either in vitro model systems [1-6] or in vivo studies utilizing a low number of animals [7-9]. In vitro models utilize monocultures of representative corneal cell populations and cannot fully evaluate potential pathologic and toxic changes that may occur in the multi-layered cornea. In vivo studies that contain small sample sizes may fail to prove significance due to low statistical power. It is desirable for in vitro research to be validated in vivo prior to routine clinical application of a new treatment. As such, ex vivo models represent a valuable bridge between in vitro and in vivo research.

EX vivo models have been used to study a variety of different ocular diseases [10-16] in different species [14, 17-19]. One important benefit of an ex vivo model is that it allows for a more adequate representation of in vivo conditions. Successful corneal ex vivo models maintain normal corneal architecture, extra-cellular matrix, and different cell populations normally present within the living cornea. Additionally, ex vivo models are financially prudent, highly reproducible, and often require less regulatory approval when compared to in vivo experiments.

To our knowledge, no ex vivo model for the equine cornea exists. The goal of this study was to develop an ex vivo equine corneal model that would be highly reproducible. Two different types of ex vivo model systems were selected for study due to their previous successful use in other animals [17, 20,21]. We sought to examine an immersion organ culture system (IC) or an air/liquid interface organ culture system (ALC). We hypothesized that ALC would be superior at preserving normal equine corneal architecture and demonstrate minimal cellular apoptosis when compared to the IC.

\section{Materials and Methods}

Approval for this study was obtained by the University of Missouri Institutional Animal Care and Use Committee. A total of seven horses donated to the University of Missouri were humanely euthanized for reasons unrelated to this study. The median age of all horses was 17 years (range 14-22 years). Breeds included 4 Quarter horses, 2 Appaloosas and 1 Belgian. Prior to euthanasia, all horses had slit-lamp biomicroscopy (SL-15 Biomicroscope, Kowa Inc, Tokyo, Japan) performed OU by a board-certified veterinary ophthalmologist (EAG) and were deemed free of anterior segment disease.

Immediately following humane euthanasia, the ocular surface was washed with a $1 \%$ dilute betadine solution. The cornea along with the surrounding 2-3 $\mathrm{mm}$ of peri-limbal sclera was harvested by making a stab incision into the sclera with a Bard-Parker \#11 blade (Aspen Surgical, Caledonia, MI) and then continuing the incision around the peri-limbal sclera with Stevens tenotomy scissors (Integra Miltex, Plainsboro, NJ). Once the cornea and surrounding sclera (corneal-scleral ring, CSR) was harvested, the CSRs were immediately 
placed into a sterile $50 \mathrm{ml}$ test tube filled with $20 \mathrm{ml}$ of modified eagle medium (Life technologies, Carlsbad, CA), placed on ice, and promptly transported to the laboratory. Once at the laboratory, the test tube was opened under a cell culture hood and the contents placed into a sterile petri dish. Any remaining peri-limbal conjunctiva was removed at that time with sterile Stevens tenotomy scissors and the CSR was again washed with a $1 \%$ betadine solution followed by copious rinsing with sterile phosphate buffer solution (PBS). A coin toss was utilized to randomly assign the CSRs into one of two culture systems (e.g. IC or ALC).

For the IC, the CSR was sterilely placed into a $50 \mathrm{ml}$ tissue culture plate (Corning Life Sciences, Corning, NY) epithelial side up. The tissue culture plate was then filled with 15 mls of MEM media supplemented with $10 \%$ fetal bovine serum, essential amino acids, penicillin, streptomycin, fungizone, sodium pyruvate and MEM vitamins. All culture media reagents were provided by Life technologies Carlsbad, California. Following application of media the CSR was visibly inspected to ensure it was completely submerged. The total culture media was changed once daily.

For the ALC, the CSR was placed onto a specially constructed corneal conformer (Figure 1) that was designed to maintain the normal corneal curvature of the equine cornea without appling any unequal force to the corneal endothelium. The CSR and conformer were placed into a $50 \mathrm{ml}$ tissue culture plate, $13 \mathrm{mls}$ of culture media was added such that the corneal endothelium was continually bathed in culture media but a $10 \mathrm{~mm}$ diameter area of axial equine cornea was exposed to air. Media was changed daily and the exposed axial cornea received one drop of media three times daily (every 8 hours) to prevent corneal desiccation.

All CSRs were cultured at $37^{\circ} \mathrm{C}$ in $5 \% \mathrm{CO}_{2}$ for 7 days. CSRs were evaluated daily for gross evidence of either bacterial or fungal contamination, and were photographed. After 7 days in culture, the CSRs from both model systems were sectioned and one half placed into $10 \%$ neutral buffered formalin for histological analysis and the other half frozen with optimal cutting medium and placed in $-80^{\circ} \mathrm{C}$ until further analysis was performed. All model system corneas were compared histologically, through TUNEL assay, and by qPCR against healthy corneas from 4 normal control horses that were humanely euthanized for reasons other than ocular disease and processed immediately after harvesting.

\section{Corneal Transparency}

Gross photography was performed daily using a Nikon D300s camera (Nikon, Melville, New York). Corneal transparency of both model systems was evaluated by placing CSRs into a sterile petri dish and then placing the sterile petri dish over a single line of 11 point Arial font. The corneas were determined to be "opaque" (lost all corneal transparency) when the font was no longer able to be read through the axial stroma [17].

\section{Histopathologic Evaluation}

Corneal sections were routinely processed for histological evaluation with haematoxylin and eosin staining. All sections were evaluated by the primary author (TM) and graded by using a scale similar to that utilized in Janin et. al where $0=$ no stromal disorganization, $1=$ weak 
stromal disorganization, $2=$ =moderate stromal disorganization, $3=$ =strong stromal disorganization [17].

\section{Real-time polymerase chain reaction detection (qPCR)}

At study end-point, sections of axial cornea weighing 1 gram or less were taken from all CSRs in both models and individually frozen at $-80^{\circ} \mathrm{C}$ until total RNA could be extracted. For RNA extraction, each sample of cornea was placed into liquid nitrogen with $500 \mu \mathrm{l}$ RLT buffer (RNeasy kit, Qiagen, Valencia, CA) and 1\% $\beta$-mercaptoethanol. This mixture was then ground into a fine powder using a mortar and pestle, placed in a QIAshredder column (RNeasy kit, Qiagen, Valencia, CA) on ice and processed according to manufacturer's instructions. All corneas had qPCR performed evaluating for a-SMA. The forward primer for a-SMA was TGGGTGACGAAGCACAGAGC and the reverse primer was CTTCAGGGGCAACACGAAGC. This procedure was performed using a Step One Plus real-time PCR system (Applied Biosystems, Carlsbad, CA, USA) utilizing a $20 \mu$ mixture containing $2 \mu \mathrm{l}$ cDNA, $2 \mu \mathrm{l}$ forward $(200 \mathrm{nM})$ and $2 \mu \mathrm{l}$ reverse $(200 \mathrm{nM})$ primer, and $10 \mu \mathrm{l}$ of $2 \mathrm{X}$ SYBR green super mix (Bio-Rad Laboratories, Hercules, CA). This master mix was performed at a universal cycle $\left(95{ }^{\circ} \mathrm{C}\right.$ for $10 \mathrm{~min}, 40$ cycles at $95{ }^{\circ} \mathrm{C}$ for $15 \mathrm{~s}$, and $60{ }^{\circ} \mathrm{C}$ for $60 \mathrm{~s})$ according to the manufacturer's instructions [22]. $\beta$-actin was used as the housekeeping gene with the forward primer being CGGCTACAGCTTCACCACCA and the reverse primer being CGGGCAGCTCGTAGCTCTTC. These primers had previously been validated on equine corneal cells $[1,4]$. The efficacy of each primer was determined by using each PCR primers expression ratio. From this, a fold change was then calculated using the $2^{-\Delta \Delta C T}$ ratio.

\section{TUNEL Analysis}

A TUNEL kit (ApopTag Plus In Situ Apoptosis Fluorescein Detection Kit; Millipore, Billerica, MA) was utilized for all TUNEL testing in accordance with manufacturer's instructions [23, 24]. Briefly, all tissue sections had an application of equilibrium buffer followed by an application of TdT enzyme which was incubated together with the corneal cryosection for 1 hour at $37^{\circ} \mathrm{C}$ in a humidified chamber. These corneal cryosections then had an application of the stop solution consisting of Apop taq R mixed with water, followed by a PBS wash. Anti-digoxigenin Rhodamine was applied and the slides were counterstained with Vectashield Mounting Medium containing DAPI (Vector labs, United Kingdom) [24]. The number of cells exhibiting apoptosis as indicated by having a red nucleoli on fluorescence were counted in $510 x$ fields per corneal cryosection and averaged for statistical analysis.

\section{Statistical Analysis}

A Mann-Whitney test was used to determine significance for all gross photography and histologic results. Statistical analysis of PCR and TUNEL data was performed using a oneway ANOVA and Tuckey's test. A P-value of equal to or less than 0.05 was considered significant. 


\section{Results \\ Corneal Transparency}

Analysis of corneal transparency was determined if A) the sample was still transparent yielding a value of 0 for statistical analysis or B) the sample was opaque (unable to clearly visualize the Arial font) and therefore given a value of 1 . At the end of the culture period all corneas in the IC had a uniform loss of corneal transparency (Figure 2). This was in stark contrast to corneas in ALC which ubiquitously maintained the natural transparent state of the equine cornea. Differences between the IC and the ALC were significant $(\mathrm{p}<0.01)$.

\section{Histological Evaluation}

Histological evaluation of all healthy control corneas yielded a grade 0 . IC corneas uniformly had complete loss of the corneal epithelium, breaks in descemet's membrane and severe corneal edema (grade 3 ) which resulted in an overall loss of the normal corneal architecture (Figure 3b). Histological evaluation of ALC corneas yielded the following: 3 receiving a grade 0 , and 4 receiving a grade 1 . For grade 1 corneas there was mild corneal edema which minimally disrupted the corneal architecture (Figure $3 \mathrm{c}$ ). Corneas from the ALC (both grade 0 and grade 1) had both their corneal epithelium and endothelium intact at the end of the culture period. A significant difference between IC corneas was detected between both controls $(\mathrm{p}<0.01)$ and ALC corneas $(\mathrm{p}<0.01)$. No significant difference between the ALC model and control group corneas was identified $(\mathrm{p}=0.073)$.

\section{Real-time PCR}

Real-time PCR was performed on all samples evaluating for a-SMA. IC corneas showed a significant increase in a-SMA levels in comparison to controls $(\mathrm{p}<0.01)$. The magnitude of this increase was levels of a-SMA nearly seven times higher than that found in healthy control corneas. Significantly increased a-SMA levels were found in IC corneas when compared to the ALC corneas ( $\mathrm{p}<0.01)$. Corneas from the ALC had higher a-SMA levels than controls, but the results were not significant $(\mathrm{p}=0.749)$ (Figure 4).

\section{TUNEL}

On TUNEL evaluation an increased level of cellular apoptosis was found in IC corneas (Figure 5b) compared to controls (Figure 5a), these results were significant $(p<0.01)$ Results of ALC corneas (Figure 5c) and control corneas showed no significant difference $(\mathrm{p}=0.899)$.

\section{Discussion}

Our data suggests that the air-liquid interface organ culture system better preserves the equine cornea when compared to the immersion condition organ culture system. We were able to adequately maintain the integrity of the equine corneal epithelium, stroma, and endothelium using the ALC with only minimal corneal edema present at study endpoint. By contrast, corneas in the IC had severe distortion of both the cellular and extracellular components of the equine cornea which resulted in a complete loss of corneal transparency and overall corneal architecture. 
Since the corneal epithelial cells are nourished through the ocular tear film, one of the main challenges of any ex vivo corneal model is mimicking the constant blinking that allows for uniform distribution of the pre-corneal tear film. Previous studies have tried to resolve this issue by either developing a mechanical means of mimicking blinking through the use of a superfusion apparatus [25]. With the use of this machine the investigator is able to maintain a desired temperature, the corneas exposure to media, and artificial tear flow all while in a sterile environment. Other investigators have developed mechanical arms allowing for the eyelid to be pulled over the cornea after a drop was administered to mimic a blink [26]. Another route of maintaining adequate nutrition to the corneal epithelium is by placing the entire model on a rocker that allows for the organ culture media to periodically flow over the entire cornea at a desired rate [20]. In the paper by Deshpande et. al it was shown that rocking media over the entire cornea allowed for an extension of the model's life by up to 4 weeks when compared to static controls that only received a topical application of media once daily. This current study differed from this afore mentioned study, as we elected to treat the air/liquid cornea multiple times a day, thereby providing more nutrition to the corneal epithelium and preventing corneal desiccation. While we cannot directly compare our study which was a week in duration and in a different animal species with the study that was 4 weeks in duration [20], on histopathologic evaluation we found similar results with regards to overall corneal architecture and thickness of the corneal epithelium.

While the IC has been utilized for human limbal cell culture models [21], our study results do not support the use of this model for a complete equine corneal ex vivo model system. The corneal stroma is maintained in a state of relative detrugesence through the corneal epithelium and endothelium providing a barrier to fluid entering into the cornea and the corneal endothelium utilizes a $\mathrm{Na}+/ \mathrm{K}+$ pump to efficiently remove fluid from the corneal storma [27]. In all IC corneas there was a relative absence of corneal epithelium, which likely resulted in the corneal stroma being constantly exposed to the organ culture media. Cottlier described that a loss of either the corneal epithelium or endothelium resulted in a $200 \%$ or $500 \%$ increase in corneal thickness, respectively [28]. The severe corneal edema we observed resulted in overall loss of gross transparency in all IC corneas. The loss of the epithelium seen in this study likely also initiated the multifaceted process of corneal wound healing. This could explain the nearly seven fold increase in a-SMA, a known cellular cytokine indicative of corneal fibroblast to myofibroblast transformation [29] when compared to controls.

A common problem with corneal ex vivo model systems is that while they include all layers of the cornea and the extracellular matrix, damage to the fragile corneal endothelium is not uncommon with time. In the ex vivo ALC model described in this paper, we noted that as with other published corneal ex vivo model systems, there was some histological evidence of endothelial damage where the corneal conformer directly contacted the corneal endothelium. However, in the axial cornea of the ALC group the corneal endothelium did not appear broken or damaged and was still attached to Descemet's membrane on histopathology. Additionally, the corneal endothelium did not have a significant positive fluorescence on TUNEL assay indicating minimal apoptosis was present at study endpoint. Our ALC model system for equine corneas appears to afford similar endothelial protection as observed in other successful ex vivo models in various species [14, 30]. 
EX vivo corneal models have been utilized to study a variety of different diseases in both veterinary and physician ophthalmology. A recent paper by Harman et. al utilized a similar ALC canine corneal model to study acute keratitis from a CHV-1 outbreak [18]. Another paper by Bhasker et. al examined the use of a bovine ex vivo model to evaluate the protective effects of nanoformulated therapeutics [19]. In physician ophthalmology there is a move to eliminate the Draize test due to animal welfare concerns [31]. As a result there is a rise in the use of ex vivo corneal models used to study the effects of different compounds on the eye [10]. Our goal is to utilize the best possible equine corneal ex vivo model to study equine corneal disease. Our laboratory currently has experiments underway evaluating the fibrotic response of ex vivo equine corneas after applying an alkaline burn. Other potential applications of the ALC model include the investigation of different topical treatments for bacterial or fungal keratitis. Finally, this equine ex vivo model could be used to determine different corneal drug toxicities prior to direct application.

Our data indicates that the IC is an inappropriate model to study the equine cornea due to rapid corneal deterioration. By contrast, the ALC preserves near normal equine corneal structure and function for 7 days. Future studies aimed at utilizing the ALC ex vivo model to study equine corneal fibrosis are currently underway by our laboratory.

\section{Acknowledgments}

The work was primarily supported from the Ruth M Kraeuchi Missouri Endowment of Ophthalmology, MU Columbia fund (RRM) and partially through the 1I01BX00357 Veteran Health Affairs Merit (RRM), Vision for Animals Foundation VAF2015-03 (TM) and RO1EY017294 National Eye Institute (RRM) grants. The authors gratefully acknowledge the support of Dr. Govindraj Anumanthan, Mr. Justin Brooke and Mr. Prashant R. Sinha.

\section{References}

1. Donnelly KS, Giuliano EA, Sharma A, Tandon A, Rodier JT, Mohan RR. Decorin-PEI nanoconstruct attenuates equine corneal fibroblast differentiation. Vet Ophthalmol. 2014; 17:162169. [PubMed: 23718145]

2. Fink MK, Giuliano EA, Tandon A, Mohan RR. Therapeutic potential of Pirfenidone for treating equine corneal scarring. Vet Ophthalmol. 2014

3. Tandon A, Tovey JC, Sharma A, Gupta R, Mohan RR. Role of transforming growth factor Beta in corneal function, biology and pathology. Curr Mol Med. 2010; 10:565-578. [PubMed: 20642439]

4. Donnelly KS, Giuliano EA, Sharm A, Mohan RR. Suberoylanilide hydroxamic acid (vorinostat): its role on equine corneal fibrosis and matrix metalloproteinase activity. Vet Ophthalmol. 2014; 17(Suppl 1):61-68. [PubMed: 25126665]

5. Pearce JW, Giuliano EA, Moore CP. In vitro susceptibility patterns of Aspergillus and Fusarium species isolated from equine ulcerative keratomycosis cases in the midwestern and southern United States with inclusion of the new antifungal agent voriconazole. Vet Ophthalmol. 2009; 12:318-324. [PubMed: 19751493]

6. Cullor JS, Mannis MJ, Murphy CJ, Smith WL, Selsted ME, Reid TW. In vitro antimicrobial activity of defensins against ocular pathogens. Arch Ophthalmol. 1990; 108:861-864. [PubMed: 2112378]

7. Mancuso LA, Lassaline M, Scherrer NM. Porcine urinary bladder extracellular matrix grafts (ACell Vet Corneal Discs) for keratomalacia in 17 equids (2012-2013). Vet Ophthalmol. 2014

8. Revold T, Abayneh T, Brun-Hansen H, Kleppe SL, Ropstad EO, Hellings RA, Sorum H. Listeria monocytogenes associated kerato-conjunctivitis in four horses in Norway. Acta Vet Scand. 2015; 57:76. [PubMed: 26552393]

9. Gallhoefer NS, Spiess BM, Guscetti F, Hilbe M, Hartnack S, Hafezi F, Pot SA. Penetration depth of corneal cross-linking with riboflavin and UV-A (CXL) in horses and rabbits. Vet Ophthalmol. 2015 
10. Spoler F, Kray O, Kray S, Panfil C, Schrage NF. The Ex Vivo Eye Irritation Test as an alternative test method for serious eye damage/eye irritation. Altern Lab Anim. 2015; 43:163-179. [PubMed: 26256395]

11. Gore DM, O'Brart D, French P, Dunsby C, Allan BD. Transepithelial Riboflavin Absorption in an Ex Vivo Rabbit Corneal Model. Invest Ophthalmol Vis Sci. 2015; 56:5006-5011. [PubMed: 26230765]

12. Walker JL, Bleaken BM, Wolff IM, Menko AS. Establishment of a Clinically Relevant Ex Vivo Mock Cataract Surgery Model for Investigating Epithelial Wound Repair in a Native Microenvironment. J Vis Exp. 2015:e52886. [PubMed: 26132117]

13. Pinilla Cortes L, Burd HJ, Montenegro GA, D'Antin JC, Mikielewicz M, Barraquer RI, Michael R. Experimental protocols for ex vivo lens stretching tests to investigate the biomechanics of the human accommodation apparatus. Invest Ophthalmol Vis Sci. 2015; 56:2926-2932. [PubMed: 26024078]

14. Castro-Combs J, Noguera G, Cano M, Yew M, Gehlbach PL, Palmer J, Behrens A. Corneal wound healing is modulated by topical application of amniotic fluid in an ex vivo organ culture model. Exp Eye Res. 2008; 87:56-63. [PubMed: 18555991]

15. Choy EP, To TS, Cho P, Benzie IF, Choy CK. Viability of porcine corneal epithelium ex vivo and effect of exposure to air: a pilot study for a dry eye model. Cornea. 2004; 23:715-719. [PubMed: 15448499]

16. Kang S, Park S, Noh H, Seo K. Fluid dynamics and intraocular pressure using venturi phacoemulsification machine in dogs ex vivo. Vet Ophthalmol. 2014

17. Janin-Manificat H, Rovere MR, Galiacy SD, Malecaze F, Hulmes DJ, Moali C, Damour O. Development of ex vivo organ culture models to mimic human corneal scarring. Mol Vis. 2012; 18:2896-2908. [PubMed: 23233791]

18. Harman RM, Bussche L, Ledbetter EC, Van de Walle GR. Establishment and characterization of an air-liquid canine corneal organ culture model to study acute herpes keratitis. J Virol. 2014; 88:13669-13677. [PubMed: 25231295]

19. Bhasker S, Kislay R, Rupinder KK, Jagat KR. Evaluation of nanoformulated therapeutics in an exvivo bovine corneal irritation model. Toxicol In Vitro. 2015; 29:917-925. [PubMed: 25683620]

20. Deshpande P, Ortega I, Sefat F, Sangwan VS, Green N, Claeyssens F, MacNeil S. Rocking media over ex vivo corneas improves this model and allows the study of the effect of proinflammatory cytokines on wound healing. Invest Ophthalmol Vis Sci. 2015; 56:1553-1561. [PubMed: 25655804]

21. Sangwan VS, Matalia HP, Vemuganti GK, Fatima A, Ifthekar G, Singh S, Nutheti R, Rao GN. Clinical outcome of autologous cultivated limbal epithelium transplantation. Indian J Ophthalmol. 2006; 54:29-34. [PubMed: 16531667]

22. Sharma A, Sinha NR, Siddiqui S, Mohan RR. Role of $5^{\prime}$ TG3'-interacting factors (TGIFs) in Vorinostat (HDAC inhibitor)-mediated Corneal Fibrosis Inhibition. Mol Vis. 2015; 21:974-984. [PubMed: 26330748]

23. Woo JE, Park WC, Yoo YH, Kim SW. The efficacy of co-treatment with suberoylanilide hydroxamic acid and mitomycin $\mathrm{C}$ on corneal scarring after therapeutic keratectomy: an animal study. Curr Eye Res. 2014; 39:348-358. [PubMed: 24401036]

24. Buss DG, Sharma A, Giuliano EA, Mohan RR. Efficacy and safety of mitomycin C as an agent to treat corneal scarring in horses using an in vitro model. Vet Ophthalmol. 2010; 13:211-218. [PubMed: 20618797]

25. Elbadawy HM, Salvalaio G, Parekh M, Ruzza A, Baruzzo M, Cagini C, Ponzin D, Ferrari S. A superfusion apparatus for ex vivo human eye irritation investigations. Toxicology in Vitro. 2015; 29:1619-1627. [PubMed: 26100225]

26. Chan KY, Cho P, Boost M. Corneal epithelial cell viability of an ex vivo porcine eye model. Clin Exp Optom. 2014; 97:337-340. [PubMed: 24438477]

27. Eghrari AO, Riazuddin SA, Gottsch JD. Overview of the Cornea: Structure, Function, and Development. Prog Mol Biol Transl Sci. 2015; 134:7-23. [PubMed: 26310146]

28. Cotlier E, Moses R. The cornea. Adler's Physiology of the Eye St Louis, MO: CV Mosby Co. 1975:38-63. 
29. Fini ME, Stramer BM. How the cornea heals: cornea-specific repair mechanisms affecting surgical outcomes. Cornea. 2005; 24:S2-s11. [PubMed: 16227819]

30. Kabosova A, Kramerov AA, Aoki AM, Murphy G, Zieske JD, Ljubimov AV. Human diabetic corneas preserve wound healing, basement membrane, integrin and MMP-10 differences from normal corneas in organ culture. Exp Eye Res. 2003; 77:211-217. [PubMed: 12873452]

31. Wilhelmus KR. The Draize eye test. Surv Ophthalmol. 2001; 45:493-515. [PubMed: 11425356] 


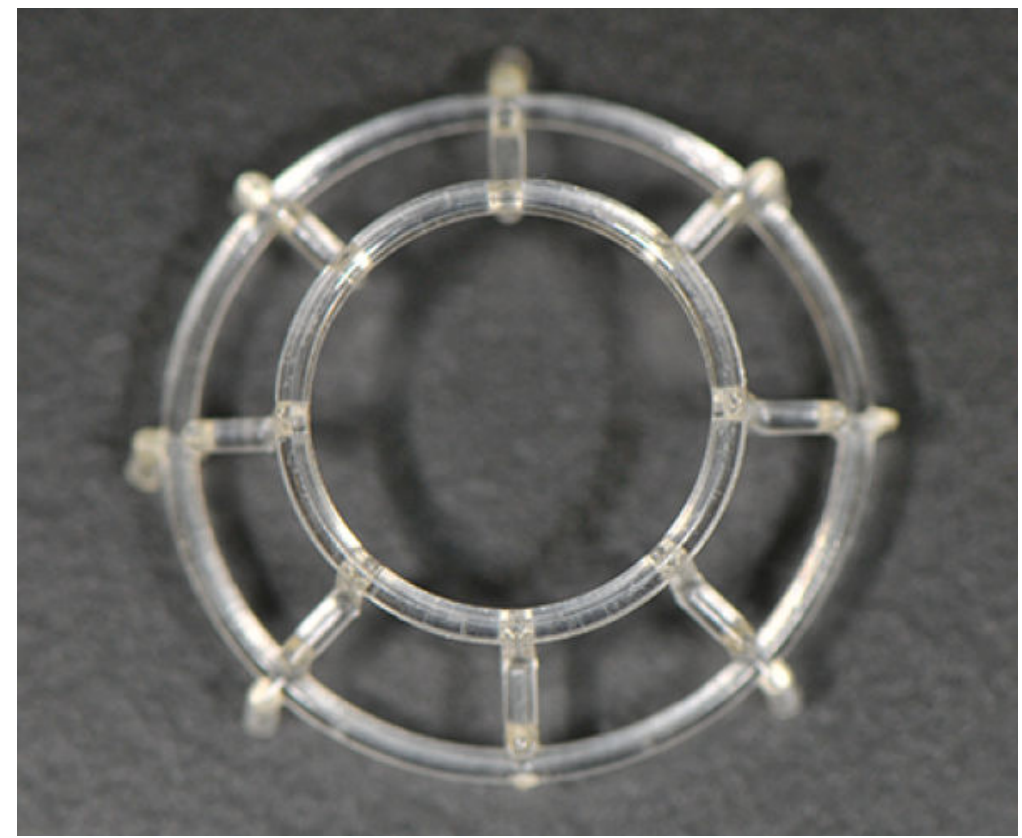

Figure 1.

Plastic conformer utilized for ALC in our study. Given the large size of the equine cornea, we found this conformer helpful in maintaining the normal corneal curvature. 


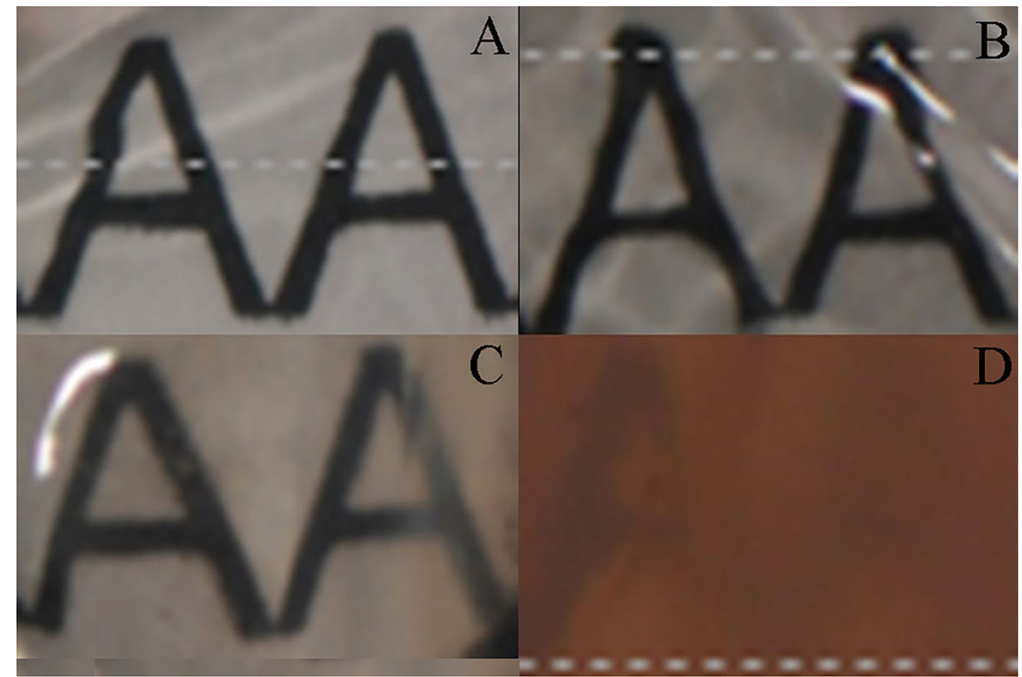

Figure 2.

Photo documentation of equine corneal transparency. (A) ALC cornea Day 0; (B) IC cornea Day 0; (C) ALC cornea Day 7; (D) IC cornea Day 7; Notice that the IC cornea has near complete loss of transparency, while the ALC cornea has good preservation of corneal transparency at study endpoint. 

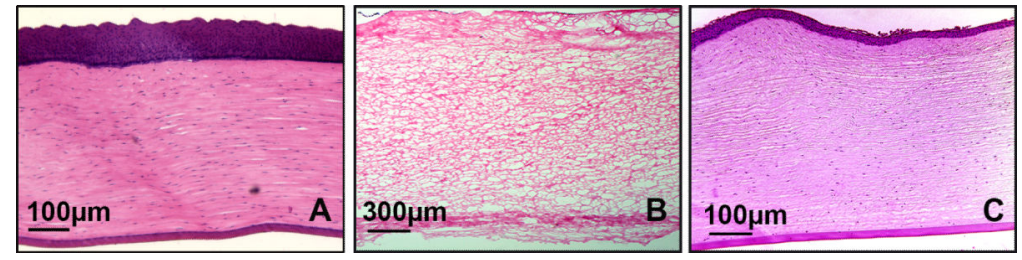

Figure 3.

Representative histological corneal images stained with $\mathrm{H} \& \mathrm{E}$ staining: (A) Healthy equine corneal control; (B) IC cornea on day 7. Complete loss of corneal epithelium along with severe stromal edema; (C) ALC cornea on day 7; corneal epithelium is still intact and only mild to moderate corneal edema is present. 


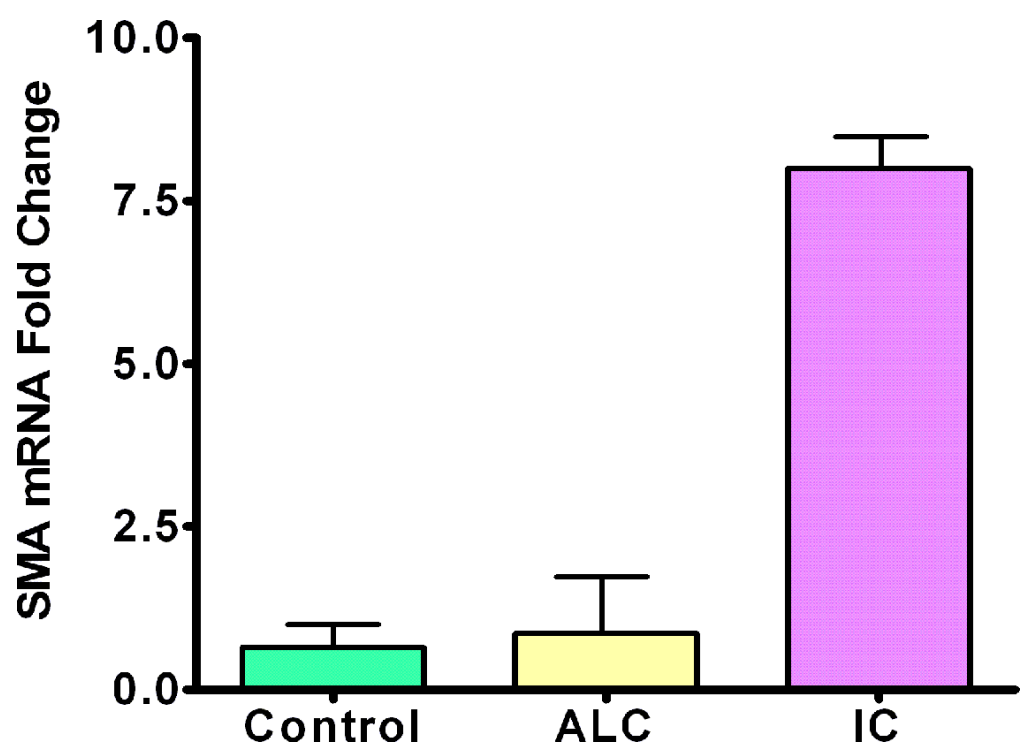

Figure 4.

Graph representing mRNA values from PCR of a-Smooth Muscle Actin (error bars indicate standard deviation). Note that both the control and ALC corneas are similar in value while the IC cornea had significantly higher aSMA values. 

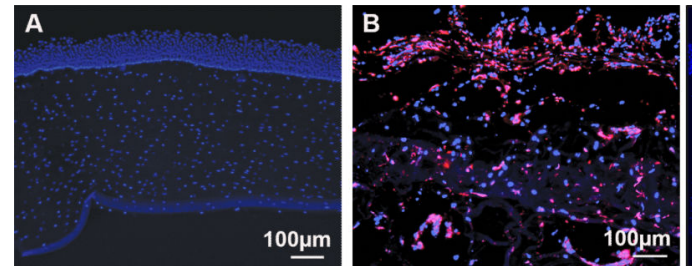

C

Figure 5.

Representative corneal images stained by TUNEL assay. (A) Healthy control; (B) IC cornea on Day 7; note the large areas of red nuclei indicating apoptotic bodies. (C) ALC cornea on Day 7; note that the cornea is minimally affected with apoptotic bodies. 